\title{
MicroRNA Profiling in Great Saphenous Vein Tissues of Patients with Chronic Venous Insufficiency
}

\author{
Chaoyi Cui, ${ }^{1,2,3}$ Guang Liu, ${ }^{1,2,3}$ Ying Huang, ${ }^{1,2,3}$ Xinwu Lu, ${ }^{1,2,3}$ Min Lu, ${ }^{1,2,3}$ \\ Xintian Huang, ${ }^{1,2,3}$ Weimin $\mathrm{Li}^{1,2,3}$ and Mier Jiang ${ }^{1,2,3}$

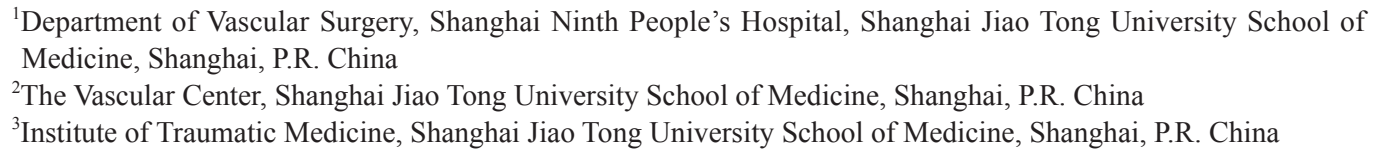

Chronic venous insufficiency $(\mathrm{CVI})$ is a common disease characterized by structural and functional abnormalities of the venous system. Until recently, the pathogenesis of CVI remains largely unknown. MicroRNAs (miRNAs) are a family of endogenous small non-coding RNAs emerged as post-transcriptional gene repressors and play essential roles in diverse pathological processes including vascular disease. However, their roles in CVI have not been elucidated. In this study, we employed oligonucleotide microarrays to perform a genome-wide miRNAs profiling in the great saphenous vein (GSV) tissues of patients with CVI. Our results revealed a total of 14 miRNAs that are expressed differentially in GSV tissues. Among them nine miRNAs were found significantly up-regulated, while five miRNAs were downregulated significantly. Real-time RT-PCR verified statistically consistent expression of three selected miRNAs (miR-34a, miR-155 and miR-202) with microarrays analysis. These three miRNAs, which were described as crucial regulators in many biological processes and vascular diseases, might also play important roles in CVI. Functional annotation of target genes of differentially expressed miRNAs via bioinformatics approaches revealed that these predicted targets were significantly enriched and involved in several key signaling pathways important for CVI, including mitogen-activated protein kinase pathways, pathways in cancer, apoptosis, and cell cycle, and p53 signaling pathways. In summary, miRNAs might involve in multiple signaling pathways contributing to the pathological processes of CVI. These data may provide fundamental insights into the molecular basis of $\mathrm{CVI}$, which may aid in designing novel approaches for prevention and treatment of this complex disease.

Keywords: bioinformatics; chronic venous insufficiency; great saphenous vein; microarray; microRNA

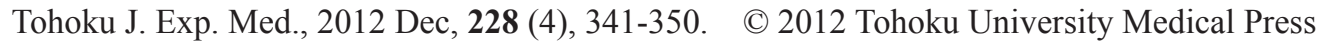

Chronic venous insufficiency (CVI) is a complex medical condition characterized by structural and functional abnormalities of the venous system, which is commonly seen in humans. CVI is manifested with a range of signs, including varicose veins, trophic skin changes and venous ulcers. It has a considerable socioeconomic impact in many countries due to its high prevalence, cost of investigations and management, deterioration of the quality of life and loss of working days (Rabe and Pannier 2010). To date, in spite of numerous studies on CVI, the etiology and pathogenesis of CVI remains largely unknown.

MicroRNAs (miRNAs) are a family of highly conserved, endogenous small non-coding RNAs (19-24nt) that post-transcriptionally repress gene expression via degradation or translational inhibition of their target mRNAs. They play important roles in nearly all physiological and pathological processes, including cell differentiation, prolifera- tion, apoptosis, cardiovascular disease, and human cancers (Bartel 2004). There is mounting evidence suggesting that miRNAs had distinct expression profiles and played crucial roles in vascular system, such as miR-126 that govern vascular integrity and angiogenesis (Wang et al. 2008), and miR-221/222 as newly found regulators for proliferation of vascular smooth muscle cell (VSMC) and neointimal hyperplasia (Liu et al. 2009). Our previous study also indicated that miRNAs might contribute to abdominal aortic aneurysm (AAA) formation by affecting multiple target genes and signaling pathways (Liu et al. 2010). Accordingly, miRNAs are known to be involved in the process of angiogenesis as well as pathogenesis of vascular diseases, serving as novel biomarkers and therapeutic targets for these diseases. However, little is known about the miRNAs profile and the functional role of miRNAs in the pathogenesis of CVI. To the best of our knowledge,

Received August 16, 2012; accepted October 20, 2012. Published online November 7, 2012; doi: 10.1620/tjem.228.341.

Correspondence: Mier Jiang, M.D., Department of Vascular Surgery, Shanghai Ninth People's Hospital, Shanghai Jiao Tong University School of Medicine, No. 639 Zhizaoju Road, Shanghai, P.R. China.

e-mail: jiangmier2011@163.com 
genome-wide miRNAs expression profiling in CVI in human has not been previously reported.

Therefore, the aim of this work is to study the potential biological functions of miRNAs in the pathogenesis of CVI. In the present work, the human miRNAs oligonucleotide microarrays (Agilent, Santa Clara, CA, USA) were used to identify differential expression of miRNAs in great saphenous vein (GSV) tissues from the patients suffering from CVI. The differentially expressed miRNAs were then selected, validated and subjected to bioinformatics analysis.

\section{Materials and Methods}

\section{Selection of patients and sample preparation}

Five male CVI patients (with mean age of $64.8 \pm 4.8$ years) were recruited to the CVI group $(n=5)$. In all these patients CVI condition was confirmed by ascending venography and duplex examinations and they were classified using the CEAP classification (the clinical-C, the etiological-E, the anatomical-A, and the pathophysiological-P) (Eklof et al. 2004). An additional five male patients (with mean age of $67.4 \pm 2.9$ years) who were going to receive lower extremity artery bypass and had no venous diseases diagnosed by extremity duplex examinations were recruited to the normal group $(n=5)$. A detailed description of CVI and normal samples is supplemented in Table 1. No significant difference in age was found between the CVI and normal groups $(p>0.05)$.

Both CVI group and normal group patients had no history of deep venous thrombosis, superficial thrombotic phlebitis, post-thrombotic syndrome, Klipple-Trenannay syndrome, May-Thurner syndrome or any other venous diseases. For this study, all the patients had signed an informed consent form, and the study was approved by the ethics committee of Shanghai Jiao Tong University School of Medicine.

In both the groups, the proximal part of the GSV near the saphenofemoral junction including the first valve $(2 \mathrm{~cm}$ of each sample) was harvested during surgical ligation and striping or artery bypass. Because cellular composition in the GSV wall is heterogeneous, a miRNAs microarrays analysis of the entire wall would be a technically challenging task. To circumvent this problem, we focused on the medial tissue, which is mainly composed of VSMC. All the samples were immediately frozen in liquid nitrogen, stored at $-80^{\circ} \mathrm{C}$ until RNA extraction. Each sample was divided into two segments, one for miRNAs microarrays study and the other for PCR verification.

\section{Total RNA extraction}

The GSV samples were homogenized with a pestle and mortar. Total RNA, including miRNAs, was extracted from the $100 \mathrm{mg}$ of the tissue by using mirVana ${ }^{\mathrm{TM}}$ RNA isolation kit (Ambion, Austin, TX, USA) following the manufacturer's instructions. The quality and quantity of each RNA sample was assessed using the Agilent 2100 Bioanalyzer (Agilent, Santa Clara, CA, USA).

\section{MiRNAs microarray profiling}

Human miRNAs microarray (Version 12.0, Agilent, Santa Clara, CA, USA) was used to identify the dysregulated miRNAs in the GSV tissues of CVI patients and control group. The RNA samples were labeled and processed according to manufacturer's recommended protocols (Wang et al. 2007). In brief, $100 \mathrm{ng}$ of total RNA was dephosphorylated with calf intestinal alkaline phosphatase, followed by denaturing with heat in the presence of dimethyl sulfoxide (DMSO). After dephosphorylation and denaturation, the total RNA was labeled with cyanine 3-pCp and then hybridized to Agilent human miRNAs microarray using the miRNAs Complete Labeling and Hyb Kit (Agilent, Santa Clara, CA, USA). Subsequently, the purified labeled miRNAs probes were hybridized to $8 \times 15 \mathrm{~K}$ human miRNAs microarrays in a rotating hybridization oven at $20 \mathrm{rpm}$ for 20 hours at $55^{\circ} \mathrm{C}$.

After hybridization of miRNAs microarray, it was washed in Gene Expression Wash Buffer 1 (Agilent, Santa Clara, CA, USA) and Gene Expression Wash Buffer 2 (Agilent, Santa Clara, CA, USA) at room temperature for 5 minutes, in rotations. The slides were then scanned with Agilent Microarray Scanner G2565A (Agilent, Santa Clara, CA, USA). The sensitivity settings were $100 \%$ and $5 \%$. Agilent Feature Extraction software (version 10.5.1, Agilent, Santa Clara, CA, USA) was used for image analysis.

Raw data were normalized and a t-test was performed to analyze the statistical significance between the two groups. The standard of significance was the corrected ratios of hybridization signal intensity between CVI and normal specimens. However, the normalized signal intensity data was generated by Quantile method using GeneSpring GX 10.0 Software (Agilent, Santa Clara, CA, USA). The

Table 1. Clinical characteristics of CVI and normal groups.

\begin{tabular}{ccccc}
\hline Patient No. & Age (years) & Male/Female & Left/Right & CEAP \\
\hline VI & 67 & $\mathrm{M}$ & $\mathrm{R}$ & $\mathrm{C}_{4 \mathrm{~S}} \mathrm{E}_{\mathrm{P}} \mathrm{A}_{\mathrm{S}} \mathrm{P}_{\mathrm{R}}$ \\
V2 & 61 & $\mathrm{M}$ & $\mathrm{R}$ & $\mathrm{C}_{4 \mathrm{~S}} \mathrm{E}_{\mathrm{P}} \mathrm{A}_{\mathrm{S}} \mathrm{P}_{\mathrm{R}}$ \\
V3 & 59 & $\mathrm{M}$ & $\mathrm{R}$ & $\mathrm{C}_{4 \mathrm{~S}} \mathrm{E}_{\mathrm{P}} \mathrm{A}_{\mathrm{S}} \mathrm{P}_{\mathrm{R}}$ \\
V4 & 66 & $\mathrm{M}$ & $\mathrm{R}$ & $\mathrm{C}_{4 \mathrm{~S}} \mathrm{E}_{\mathrm{P}} \mathrm{A}_{\mathrm{S}} \mathrm{P}_{\mathrm{R}}$ \\
V5 & 71 & $\mathrm{M}$ & $\mathrm{R}$ & $\mathrm{C}_{4 \mathrm{~S}} \mathrm{E}_{\mathrm{P}} \mathrm{A}_{\mathrm{S}} \mathrm{P}_{\mathrm{R}}$ \\
C1 & 64 & $\mathrm{M}$ & $\mathrm{R}$ & \\
$\mathrm{C} 2$ & 69 & $\mathrm{M}$ & $\mathrm{R}$ & \\
$\mathrm{C} 3$ & 71 & $\mathrm{M}$ & $\mathrm{R}$ & \\
$\mathrm{C} 4$ & 65 & $\mathrm{M}$ & $\mathrm{R}$ & \\
$\mathrm{C} 5$ & 68 & $\mathrm{M}$ & $\mathrm{R}$ & \\
\hline
\end{tabular}

$\mathrm{C}=$ normal group $; \mathrm{V}=\mathrm{CVI}$ group

Left $=$ left limb; Right $=$ right limb 
miRNAs expressions were considered significantly different if their ratios were more than 1.5 -fold or less than 0.5 -fold $(p<0.05)$. Hierarchical clustering was performed to distinguish the expression patterns among different miRNAs and samples.

\section{Real-time RT-PCR for miRNAs expression}

To validate the results of miRNAs microarrays, parts of differentially expressed miRNAs (miR-34a, miR-155 and miR-202) were selected for further verification by real-time reverse transcriptionpolymerase chain reaction (RT-PCR). Expression of miRNAs was assayed using stem-loop RT followed by PCR analysis as previously described (Chen et al. 2005). Primers for RT-PCR were synthesized by Invitrogen (USA). The reverse transcription of RNA was conducted using the miScript Reverse Transcription Kit. The cDNA serves as the template for real-time PCR analysis using a miScript Primer Assay in combination with the miScript SYBR Green PCR Kit (Qiagen, Germany). Protocols were followed according to the manufacturer's instructions. The fold change for each miRNAs was calculated using the comparative $\mathrm{Ct}\left(2^{-\triangle 4 \mathrm{Ct}}\right)$ method (Livak and Schmittgen 2001) with U6 small nuclear RNA as the endogenous control. Expression analysis was performed in triplicate for each sample.

\section{Prediction of target genes}

The online database miRBase (http://www.mirbase.org/) integrate the database of published miRNAs sequences and annotation, the services of naming novel miRNAs prior to publication, and the targets database and pipeline. The miRBase Targets database, which aims to provide a more extensive target prediction aggregation service, currently links miRNAs to targets gene predicted by MicroCosm, TargetScan and Pictar. The target genes of differentially expressed miRNAs were predicted in this database. Furthermore, the predicted genes were subjected to search across the UniGene (http:// www.ncbi.nlm.nih.gov/unigene) to define whether they had a potential function in the vascular tissues of human. The intersections of predicted genes in miRBase and genes associated with vascular tissues in UniGene were considered as putative target candidates.

\section{Bioinformatics analysis of miRNAs-targeted genes}

Gene Ontology (GO) analysis was applied to analyze the main function of the target genes according to the Gene Ontology, which is the key functional classification of NCBI. Within the significant category, the enrichment $\operatorname{Re}$ was given by: $\operatorname{Re}=\left(n_{\mathrm{f}} / \mathrm{n}\right) /\left(\mathrm{N}_{\mathrm{f}} / \mathrm{N}\right)$ where $\mathrm{n}_{\mathrm{f}}$ is the number of differential genes within the particular category, $n$ is the total number of genes within the same category, $\mathrm{N}_{\mathrm{f}}$ is the number of differential genes in the entire microarray and $\mathrm{N}$ is the total number of genes in the microarray (Schlitt et al. 2003). Afterward, we built certain MicroRNA-Go-Network, according to the relationships of significant GOs and genes and the relationships of miRNAs and genes.

Similarly, Pathway analysis was used to find out the significant pathways of the target genes according to Kyoto Encyclopedia of Genes and Genomes (KEGG) pathway database (http://www.genome. $\mathrm{jp} / \mathrm{kegg} /$ ). The enrichment Re was calculated like the equation above (Draghici et al. 2007). Consequently, the Path-Net, which was the interaction net of the significant pathways of the differential expression genes, was built according to the interaction among pathways of the KEGG database to find the interaction among the significant pathways directly and systemically (Yi et al. 2006).

\section{Statistical analysis}

All the results were expressed as mean \pm standard deviation (S.D.). Difference between CVI and normal groups was assessed by Student's $t$ test using SPSS 13.0. Fisher's exact test and $\chi^{2}$ test were used to classify the GO category and the pathway analysis. The threshold of significance was defined by $P$ value and the false discovery rate (FDR).

\section{Results}

\section{Expression profiles of miRNAs in CVI and normal groups}

After normalization of the raw data, the expression profiles of miRNAs were found to be significantly different between these two groups as shown in Fig. 1. Using strict criteria that only miRNAs undergo alternations if their ratios were more than 1.5 -fold or less than 0.5 -fold $(p<$ 0.05 ) were considered as differentially expressed candidates. A total number of 14 miRNAs were identified as listed in Table 2. Among them, nine up-regulated miRNAs were found, while five down-regulated miRNAs were identified in CVI group as compared to that in normal group.

\section{Validation of the microarray data by real-time RT-PCR}

We selected three miRNAs that displayed either an increase or decrease in expression (miR-34a, miR-155 and miR-202) to validate with real-time RT-PCR. These three miRNAs have been described as crucial regulators in many biological processes and vascular diseases (Martin et al. 2007; Chen and $\mathrm{Hu}$ 2012; Schrauder et al. 2012) and thus they might also play important roles in CVI condition. As expected, the relative fold changes in miRNAs expression as determined by real-time RT-PCR were in agreement with microarrays results, as shown in Fig. 2. All real-time RT-PCR data were consistent with that of the microarrays, suggesting that the data obtained from the miRNAs microarrays accurately reflected miRNAs expression.

\section{Prediction of target genes and bioinformatics analysis of their functions}

We searched for potential target genes of the 14 differentially expressed miRNAs in miRBase Targets database, which currently links miRNAs to target genes predicted by different computational prediction algorithms (MicroCosm, TargetScan and Pictar). Using consensus targets selected by at least two prediction programs, we identified 2417 target genes at first. Then, these genes were subjected to search across the UniGene database to define whether they are human vascular tissue-specific expression genes. As a result, the intersection of 1318 genes in miRBase and UniGene were considered as putative target genes.

These target genes were further submitted to the GO database for GO annotation and enrichment analysis. In this study, we chose only GOs that had a $p$-value $<0.01$ and a FDR $<0.05$. The high-enrichment GOs targeted by upregulated miRNAs were response to blood vessel endothelial cell migration, collagen biosynthetic process, programmed cell death and regulation of actin cytoskeleton 


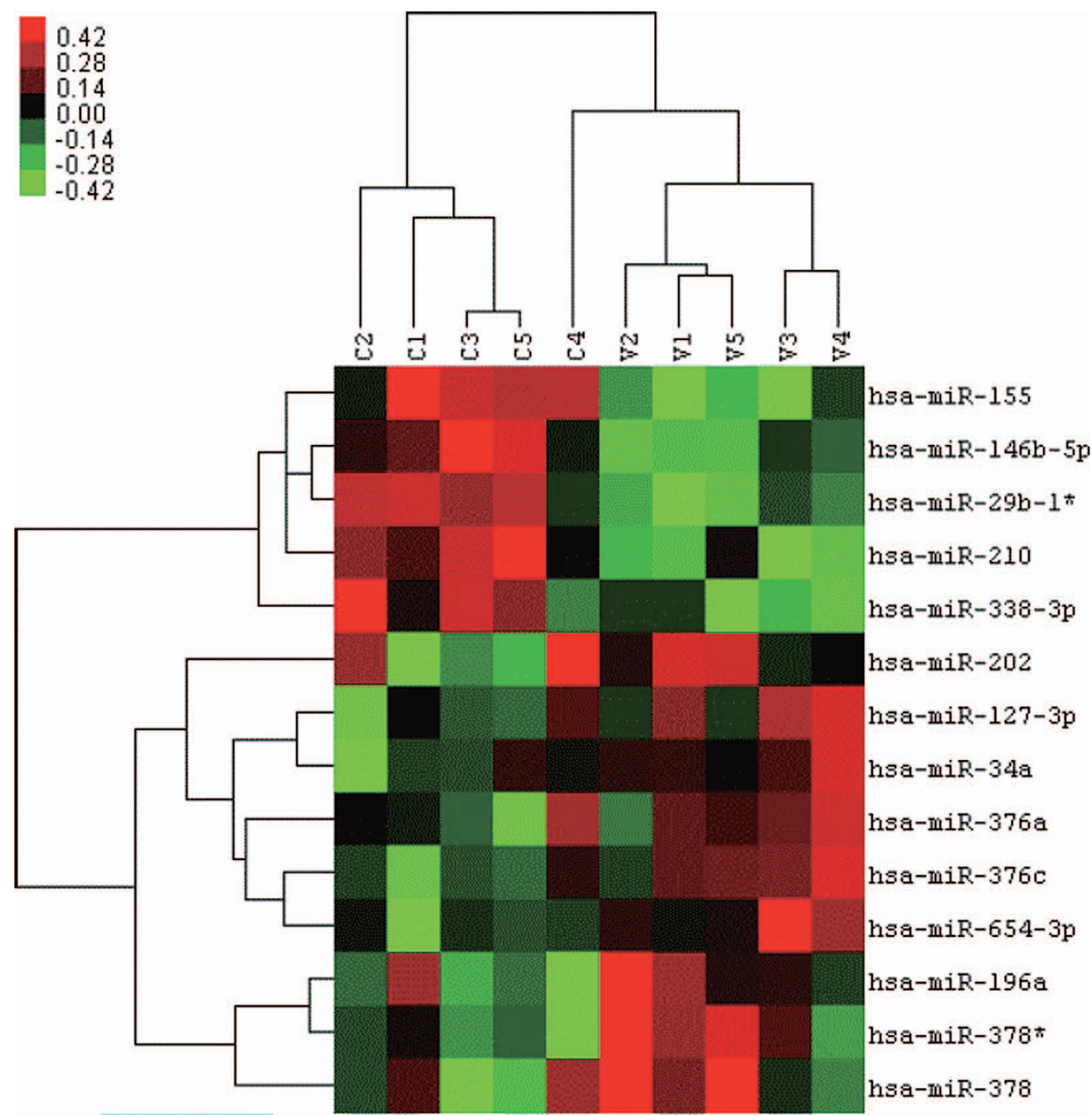

Fig. 1. Hierarchical clustering analysis of miRNAs expression of GSV tissues in CVI and normal group. MiRNAs and samples are presented in rows and columns, respectively. Colors indicate relative signal intensities; red and green colors indicate up-regulated and down-regulated miRNAs, respectively. $\mathrm{C}=$ normal group; $\mathrm{V}=\mathrm{CVI}$ group.

Table 2. The list of differentially expressed miRNAs in CVI tissues.

\begin{tabular}{lll}
\hline \multicolumn{1}{c}{ miRNAs } & $p$-value & Fold change \\
\hline $\begin{array}{l}\text { Upregulated miRNAs } \\
\text { hsa-miR-378* }\end{array}$ & 0.003283 & 2.04 \\
hsa-miR-378 & 0.007671 & 2 \\
hsa-miR-376c & 0.008273 & 1.84 \\
hsa-tniR-202 & 0.035724 & 1.8 \\
hsa-miR-127-3p & 0.022648 & 1.71 \\
hsa-miR-654-3p & 0.014088 & 1.65 \\
hsa-nliR-376a & 0.043922 & 1.6 \\
hsa-miR-196a & 0.01696 & 1.55 \\
hsa-miR-34a & 0.028176 & 1.51 \\
Dowm regulated miRNAs & & \\
hsa-miR-29b-1 * & 0.004918 & 0.49 \\
hsa-nliR-210 & 0.01691 & 0.48 \\
hsa-miR-155 & 0.012678 & 0.48 \\
hsa-miR-338-3p & 0.041149 & 0.47 \\
hsa-miR-146b-5p & 0.04433 & 0.3 \\
\hline
\end{tabular}

organization. In contrast, significant GOs corresponding to down-regulated miRNAs appeared to be actin cytoskeleton reorganization, vein smooth muscle contraction and positive regulation of anti-apoptosis (Fig. 3). The MicroRNAGo-Network was established as shown in Fig. 4. The center of the network was represented by degree. The term degree referred to the contribution of one miRNAs to the GOs around or the contribution one GO to the miRNAs around. The key miRNAs and genes in the network always had the biggest degrees (Table 3 and 4).

A separate functional analysis by the KEGG pathway database showed these target genes were significantly enriched in several key signaling pathways with $p$-value $<0.01$ and FDR $<0.05$. Mitogen-activated protein kinase (MAPK) pathways, pathways in cancer, apoptosis, cell cycle and p53 signaling pathways were abundant among the significantly enriched pathways (Table 5). The Path-Net, which could summarize the pathways interaction of differential expression genes under diseases were showed in Fig. 5 .

\section{Discussion}

Although risk factors for CVI such as aging, obesity, 


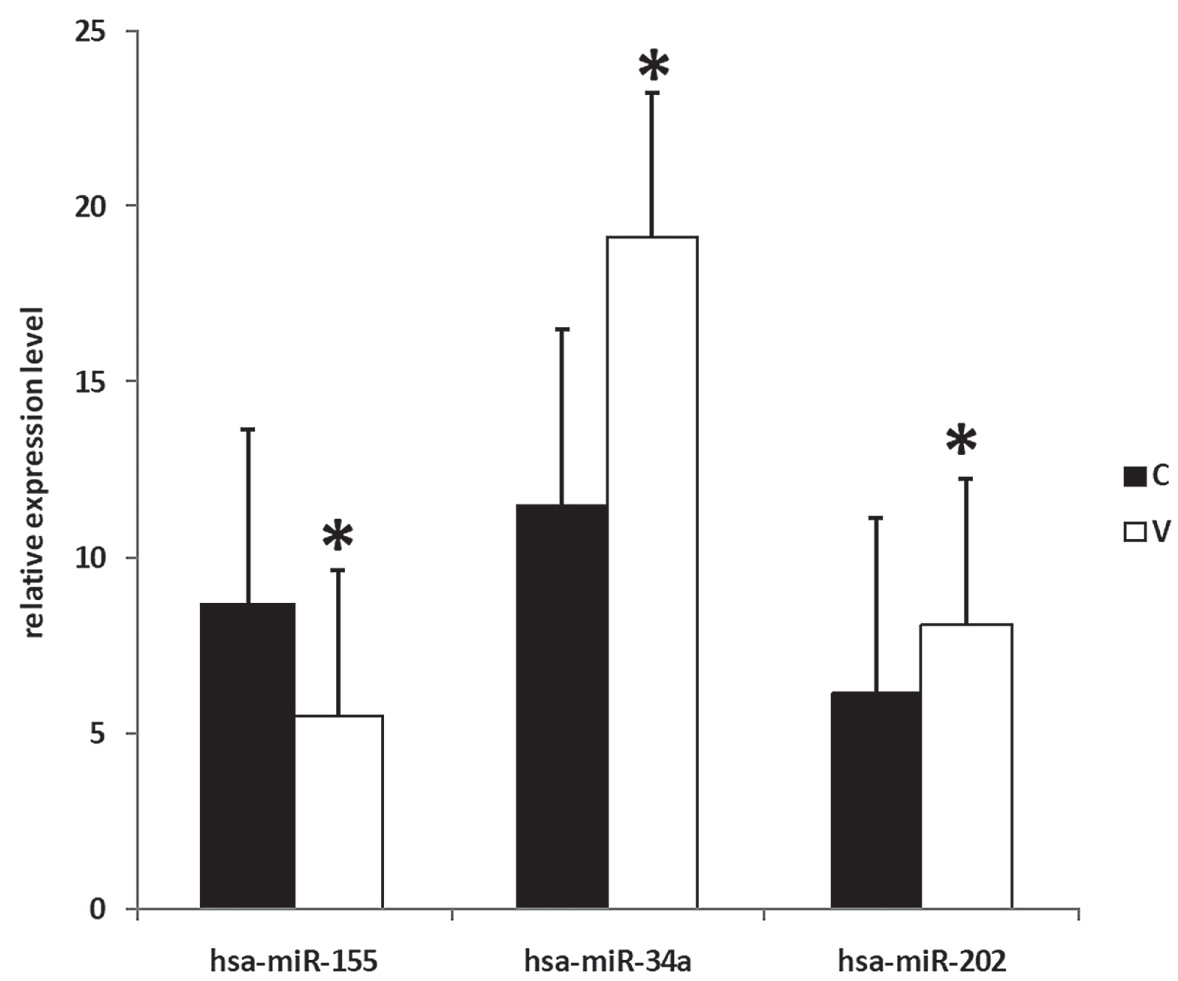

Fig. 2. Validation of microarrays data using real-time RT-PCR.

Three commonly changed miRNAs are listed on the $\mathrm{x}$-axis, and the $\mathrm{y}$-axis refers to the relative expression levels. *Significant difference between CVI and normal group. $(p<0.05)$. $\mathrm{C}=$ normal group; $\mathrm{V}=\mathrm{CVI}$ group.

pregnancy, and family history have been identified (Ruckley et al. 2002; Carpentier et al. 2004), the molecular mechanisms underlying the pathogenesis of this disease remains vague. Various theories, such as the hemodynamic alteration causing venous hypertension (Bergan et al. 2008), the incompetence of the saphenous venous valves (Duran et al. 2000), the leukocyte-trapping hypothesis (Korthuis and Unthank 2000) and the weak vein wall hypothesis (Meissner et al. 2007) had been put forward to explain the pathogenesis of varicose veins and CVI. However, these theories might remain some uncertainties and had been conflicting with each other. In recent times, more and more researchers have focused on the genetic aspect in CVI. Yin et al. detected the disease-related genes and confirmed down-regulation of Desmuslin in abnormal veins (Yin et al. 2006). Using complementary deoxyribonucleic acid (cDNA) microarrays, Lee et al. also found 82 genes that were up-regulated in varicose vein (Lee et al. 2005). However, there has been little information reported about the gene regulation of miRNAs concerning the etiologic process of CVI formation.

In this study, we evaluated the miRNAs expression profile in GSV tissues of CVI patients and normal group. Our profiling data revealed that several miRNAs were differentially expressed, with nine up-regulated and five downregulated miRNAs in CVI. Furthermore, three differentially expressed miRNAs (miR-34a, miR-155 and miR-202) were selected for validation by real-time RT-PCR. These
PCR data were in line with microarray results, thus partially verifying the reliability of the profiling results.

Among the dysregulated miRNAs (Table 2), the downregulated miR-155 might be involved in inflammatory responses during various vascular diseases. A series of studies have revealed that angiotensin II (Ang II) can induce the expression of adhesion molecules in the vascular wall and initiate the process of vascular inflammatory response (Tummala et al. 1999; Libby 2002). Ang II, the principal effector molecule of the renin-angiotensin system, shows its biological activity mainly via binding to Ang II type 1 receptor (AT1R). A study by Martin and coworkers demonstrated that AT1R and miR-155 were co-expressed in endothelial cells and VSMC and that miR-155 translationally repressed the expression of AT1R (Martin et al. 2007). Interestingly, the Ang II receptor-associated protein (AGTRAP) was also predicted to be target gene of miR-155 in our study. And miR-155 was emerging target of a broad range of inflammatory mediators and regulated lymphoid and myeloid cell functions (Rodriguez et al. 2007; Vigorito et al. 2007; O'Connell et al. 2008). As inflammation is a key event for the initiation and progression of CVI, we may predict that miR-155 might be an important modulator of vessel remodeling in this disease.

In addition, miR-378 and miR-210 have been demonstrated to be pro-angiogenic miRNAs in tumor and in endothelial cell (Yang et al. 2005; Chen et al. 2008). Angiogenesis is an essential mechanism for the mainte- 

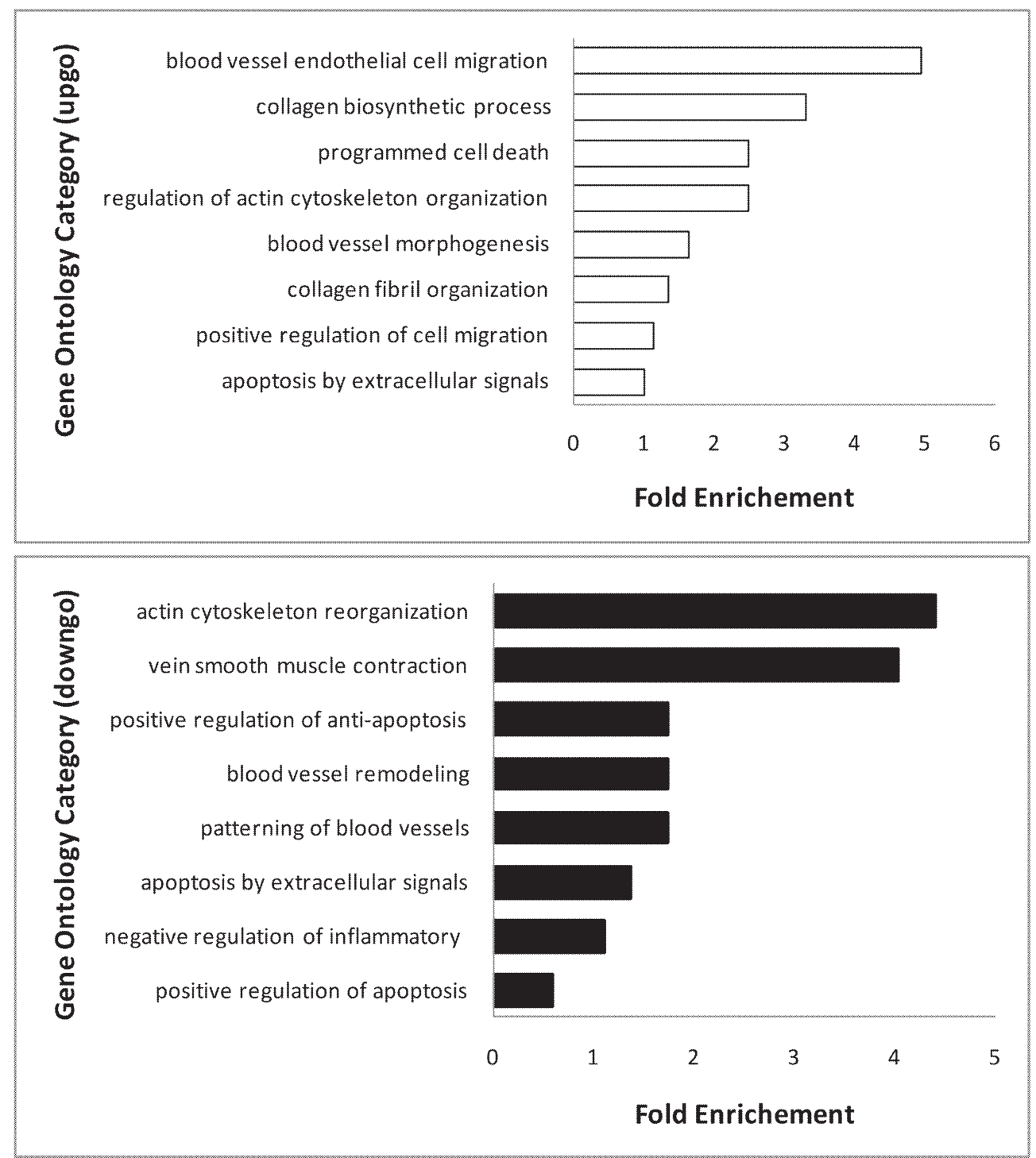

Fig. 3. Function enrichment of putative miRNAs targets.

The upper chart composes the GOs targeted by up-regulated miRNAs, and the lower chart composes the GOs targeted by down-regulated miRNAs. The vertical axis is the GO category and the horizontal axis is the enrichment of GOs.

Table 3. The degree of part miRNAs in the MicroRNAGO-Network (Top 5).

\begin{tabular}{lcc}
\hline miRNAs & style & degree \\
\hline hsa-miR-202 & up & 27 \\
hsa-miR-34a & up & 25 \\
hsa-miR-376c & up & 18 \\
hsa-rniR-155 & down & 15 \\
hsa-nliR-376a & up & 15 \\
\hline
\end{tabular}

nance of adequate blood supply to the tissues in the process of diseases such as ischemic heart disease, cancer and atherosclerosis (Zhang 2010). Apart from these angiogenesisrelated miRNAs, miR-34a regulated a plethora of target genes, which were involved in cell cycle, apoptosis, differentiation and cellular development (Chen and Hu 2012).
Table 4. The degree of part GOs in the MicroRNA-GONetwork (Top 5).

\begin{tabular}{lc}
\hline \multicolumn{1}{c}{ GO name } & degree \\
\hline Apoptosis & 10 \\
Negative regulation of cell proliferation & 10 \\
Positive regulation of cell proliferation & 9 \\
Induction of apoptosis by extracellular signals & 7 \\
Actin cytoskeleton reorganization & 6 \\
\hline
\end{tabular}

What's more, miR-202, which were known to be involved in self-renewal and tumorigenicity of breast cancer cells, might be a new blood-based biomarkers for early stage breast cancer detection (Schrauder et al. 2012).

In order to gain insights into the function of these miRNAs, bioinformatics analysis (including prediction of 


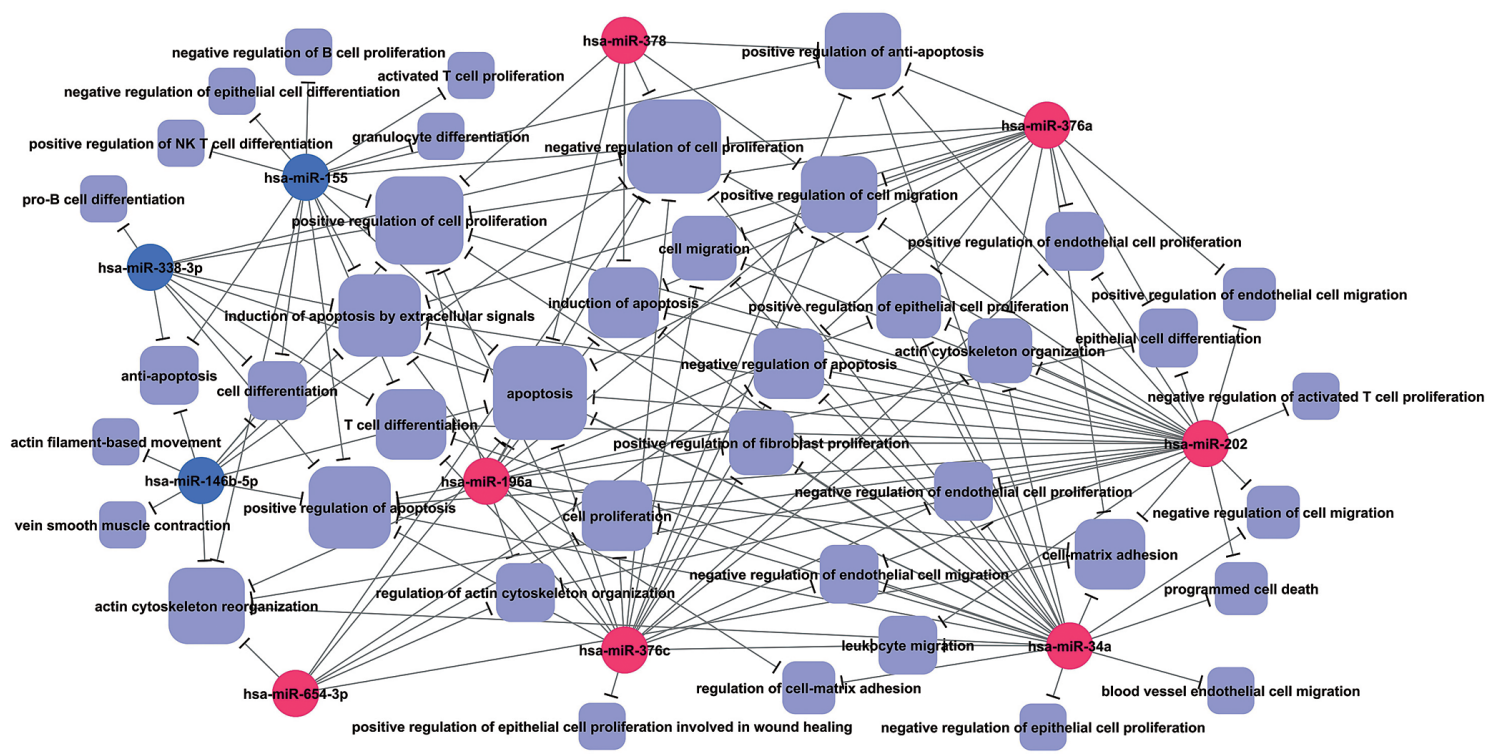

Fig. 4. The MicroRNA-Go-Network.

The red circle represents up-regulated miRNAs, while the blue circle represents down-regulated miRNAs. The shape of square represents the GO function of target genes. The relationship of miRNAs and target GO function is represented by one edge. The center of the network is represented by degree. The term degree refers to the contribution of one miRNAs to the GOs around or the contribution one GO to the miRNAs around. The key miRNAs and GOs in the network always have the biggest degrees.

target genes, GO analysis and Pathway analysis) were applied to their target genes. The high-enrichment GOs mostly focused on blood vessel endothelial cell migration, collagen biosynthetic process, programmed cell death, actin cytoskeleton reorganization and vein smooth muscle contraction. These biological processes might induce alterations to the contents of elastin, collagen and VSMC in tunica media, which play important role in vessel remodeling in CVI (Lowell et al. 1992). For the first time, our research revealed that miR-202 and miR-196a were up-regulated in the GSV tissues of CVI patients. Furthermore, these two miRNAs were predicted to target collagen-related genes (COL1A2 and COL3A1) in miRBase. As a result of a little speculation, we have come to the assumption that the upward expression of miR-202 and miR-196a may lead to the disorders in metabolism of collagen in the GSV wall and contribute to the pathogenesis of CVI. In addition, matrix metalloproteinases (MMPs), a family of proteases as major determinants of venous tissue remodeling, were involved in the continuous turnover of collagen, elastin and other proteins of extracellular matrix (ECM). The effects of MMPs on ECM degradation might result in significant venous tissue remodeling and structural changes in the vein wall, leading to venous dilation and valve dysfunction (Raffetto and Khalil 2008). In the study, miR-202 (target to MMP11) and miR-146b-5p (target to MMP16) might be the regulatory miRNAs associated with MMPs regulation in CVI condition. However, these interpretations need to be additionally validated with further studies.

Based on the MicroRNA-Go-Network, GOs concerning apoptosis (10 degrees) were seemed to dominate the significant GO categories. MiR-202 (27 degrees) and miR$34 \mathrm{a}$ ( 25 degrees) might be in the central position of regulation. Apoptosis of vascular cells was a prominent feature of blood vessel remodeling that occurs during normal development and many disorders of the vessel wall. It had been demonstrated that the overall number of apoptotic cells and activity were reduced in varicose compared with non-varicose veins (Bujan et al. 2000; Ascher et al. 2001). Ducasse et al. provided evidence of decreased programmed cell death in the medial layer of human primary varicose veins (Ducasse et al. 2008). In our research, CASP3, the most important and final executant of apoptosis, was predicted to be target gene of miR-202. The over expression of miR202 may lead to reduction of CASP3, which may explain the dysregulation of VSMC apoptosis in CVI. Since our results seem somewhat more general, the assumption we made for the function of miRNAs may be verified by profound research.

The KEGG Pathway analysis showed a significant change with several signaling pathways in the CVI group compared with the normal group. The Path-Net based on the interaction net of the significant pathways of the differential expression genes, further displayed MAPK signaling pathway (40 degrees) as a core pathway in the net. Emerging evidence supported the notion that the MAPK signaling pathway was a common pathway in many vascular diseases. A miRNAs profile comparison between thoracic aortic dissection and normal thoracic aorta indicated that miR-30 families were likely to play a role in MAPK signaling pathway (Liao et al. 2011). Moreover, Raffetto et al. (2006) suggested the importance of the MAPK signal- 


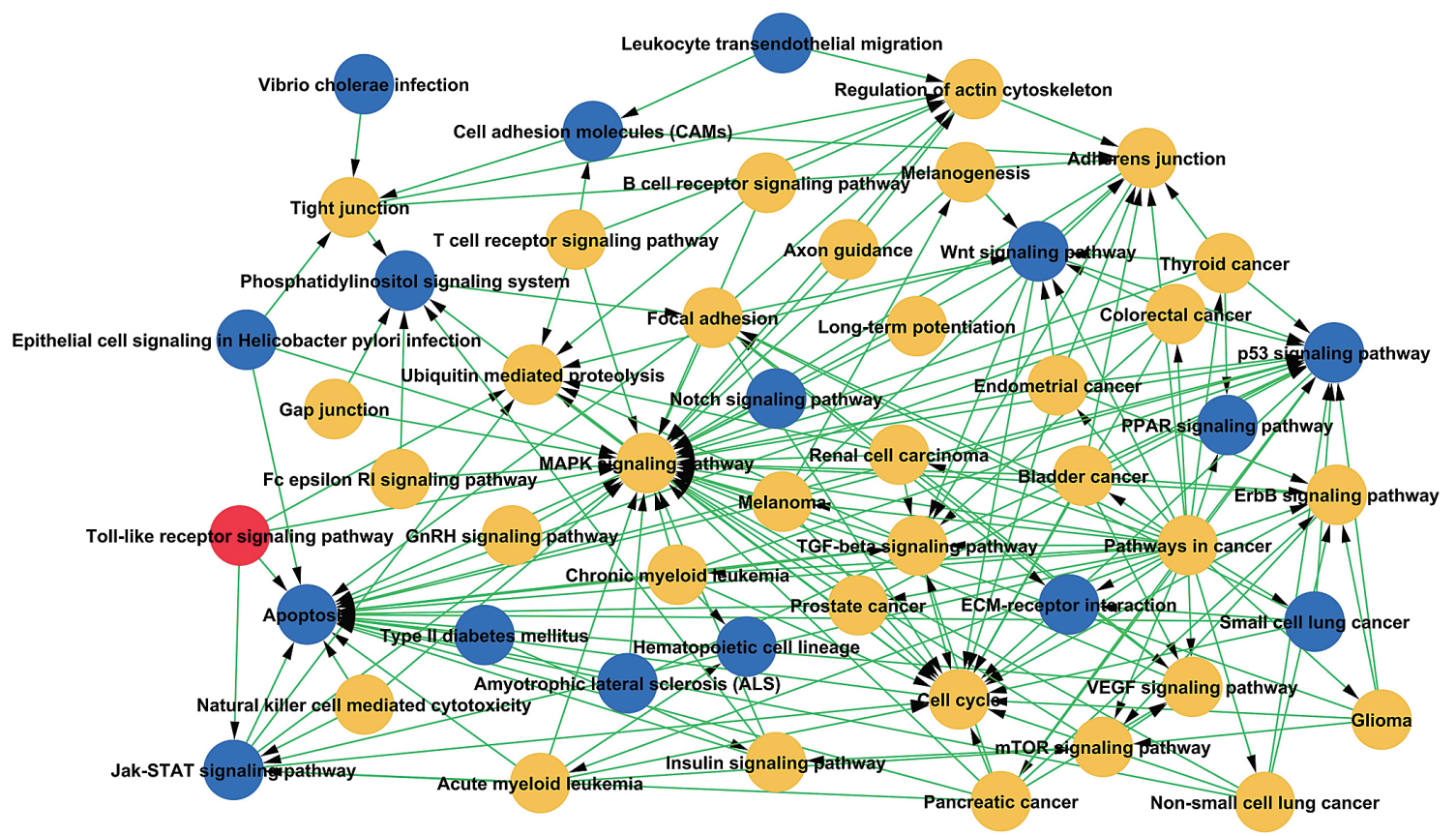

Fig. 5. The Path Net.

The red circle represents up-regulated pathways, the blue circle represents down-regulated pathways and the yellow circle represents both up-regulated and down-regulated pathways. The relationship of the pathways is represented by an arrow. The center of the network is represented by degree. The term degree refers to the number of interaction relationship of pathways. The key pathway in the network always has the biggest degrees.

Table 5. The degree of part pathways in the Path-Net (degree $\geq 10$ ).

\begin{tabular}{lcccc}
\hline \multicolumn{1}{c}{ pathway name } & degree & outdegree $^{\mathrm{a}}$ & indegree $^{\mathrm{b}}$ & $p$-value $^{-}$ \\
\hline MAPK signaling pathway & 40 & 4 & 36 & $1.64 \mathrm{E}-06$ \\
Pathways in cancer & 27 & 27 & 0 & $6.62 \mathrm{E}-16$ \\
Apoptosis & 22 & 0 & 22 & 0.00887 \\
Cell cycle & 21 & 2 & 19 & 0.00561 \\
P53 signaling pathway & 17 & 2 & 15 & $2.34 \mathrm{E}-09$ \\
Wnt signaling pathway & 12 & 6 & 6 & $5.67 \mathrm{E}-06$ \\
Adherens junction & 11 & 2 & 9 & $8.83 \mathrm{E}-10$ \\
TGF-beta signaling pathway & 11 & 4 & 7 & 0.00077 \\
Focal adhesion & 10 & 6 & 4 & 0.00059 \\
\hline
\end{tabular}

outdegree $^{\mathrm{a}}=$ the number of downstream pathway of a certain pathway

indegree $^{b}=$ the number of upstream pathway of a certain pathway

ing pathway in regulating venous ulcer fibroblasts proliferation and the inhibitory effects of wound fluid on this pathway. In addition, several genes involved in MAPK signaling pathway, such as MAP3K9 and MAP3K10, were validated to be the target of miR-34a (Tivnan et al. 2011). However, more work is needed to establish the molecular networks that consisted with miRNAs and multiple signaling pathways in this disease.

In summary, our research provided a holistic view of miRNAs expression profiles for the first time in CVI patients. The bioinformatics analysis of these data indicated that miRNAs and target genes probably involved in multiple signaling pathways in pathological processes of
CVI. Because we only focused on the $\mathrm{C} 4$ patients in the study (Table 1), future studies evaluating miRNAs in other $\mathrm{C}$ classification will be required to elucidate the role of miRNAs in the spectrum of CVI. Although the exact function of these miRNAs and genes remains to be confirmed, we believe the present research will provide fundamental novel insights into the molecular basis of CVI, which may aid in designing novel approaches for prevention and treatment of this complex disease condition.

\section{Acknowledgments}

This research was supported by the National Natural Science Foundation of China (No. 81170292 and No. 81100223) 
and the Doctoral Innovation Foundation of Shanghai Jiao Tong University School of Medicine (No. BXJ 201129).

\section{Conflict of Interest}

All authors have no conflict of interest in this study.

\section{References}

Ascher, E., Jacob, T., Hingorani, A., Tsemekhin, B. \& Gunduz, Y. (2001) Expression of molecular mediators of apoptosis and their role in the pathogenesis of lower-extremity varicose veins. J. Vasc. Surg., 33, 1080-1086.

Bartel, D.P. (2004) MicroRNAs: genomics, biogenesis, mechanism, and function. Cell, 116, 281-297.

Bergan, J.J., Pascarella, L. \& Schmid-Schonbein, G.W. (2008) Pathogenesis of primary chronic venous disease: Insights from animal models of venous hypertension. J .Vasc. Surg., 47, 183-192.

Bujan, J., Jimenez-Cossio, J.A., Jurado, F., Gimeno, M.J., Pascual, G., Garcia-Honduvilla, N., Dominguez, B. \& Bellon, J.M. (2000) Evaluation of the smooth muscle cell component and apoptosis in the varicose vein wall. Histol. Histopathol., 15, 745-752.

Carpentier, P.H., Maricq, H.R., Biro, C., Poncot-Makinen, C.O. \& Franco, A. (2004) Prevalence, risk factors, and clinical patterns of chronic venous disorders of lower limbs: a population-based study in France. J. Vasc. Surg., 40, 650-659.

Chen, C., Ridzon, D.A., Broomer, A.J., Zhou, Z., Lee, D.H., Nguyen, J.T., Barbisin, M., Xu, N.L., Mahuvakar, V.R., Andersen, M.R., Lao, K.Q., Livak, K.J. \& Guegler, K.J. (2005) Real-time quantification of microRNAs by stem-loop RT-PCR. Nucleic Acids Res., 33, e179.

Chen, F. \& Hu, S.J. (2012) Effect of microRNA-34a in cell cycle, differentiation, and apoptosis: a review. J .Biochem. Mol. Toxicol., 26, 79-86.

Chen, J.F., Murchison, E.P., Tang, R., Callis, T.E., Tatsuguchi, M., Deng, Z., Rojas, M., Hammond, S.M., Schneider, M.D., Selzman, C.H., Meissner, G., Patterson, C., Hannon, G.J. \& Wang, D.Z. (2008) Targeted deletion of Dicer in the heart leads to dilated cardiomyopathy and heart failure. Proc. Natl. Acad. Sci. USA, 105, 2111-2116.

Draghici, S., Khatri, P., Tarca, A.L., Amin, K., Done, A., Voichita, C., Georgescu, C. \& Romero, R. (2007) A systems biology approach for pathway level analysis. Genome Res., 17, 15371545.

Ducasse, E., Giannakakis, K., Speziale, F., Midy, D., Sbarigia, E., Baste, J.C. \& Faraggiana, T. (2008) Association of primary varicose veins with dysregulated vein wall apoptosis. Eur. $J$ .Vasc. Endovasc. Surg., 35, 224-229.

Duran, W., Pappas, P.J. \& Schmid-Schonbein, G.W. (2000) Microcirculatory inflammation in chronic venous insufficiency: current status and future directions. Microcirculation, $\mathbf{7}$, S49-58.

Eklof, B., Rutherford, R.B., Bergan, J.J., Carpentier, P.H., Gloviczki, P., Kistner, R.L., Meissner, M.H., Moneta, G.L., Myers, K., Padberg, F.T., Perrin, M., Ruckley, C.V., Smith, P.C. \& Wakefield, T.W. (2004) Revision of the CEAP classification for chronic venous disorders: consensus statement. $J$. Vasc. Surg., 40, 1248-1252.

Korthuis, R.J. \& Unthank, J.L. (2000) Experimental models to investigate inflammatory processes in chronic venous insufficiency. Microcirculation, 7, S13-22.

Lee, S., Lee, W., Choe, Y., Kim, D., Na, G., Im, S., Kim, J., Kim, M. \& Cho, J. (2005) Gene expression profiles in varicose veins using complementary DNA microarray. Dermatol. Surg., 31, 391-395.

Liao, M., Zou, S., Weng, J., Hou, L., Yang, L., Zhao, Z., Bao, J. \& Jing, Z. (2011) A microRNA profile comparison between thoracic aortic dissection and normal thoracic aorta indicates the potential role of microRNAs in contributing to thoracic aortic dissection pathogenesis. J. Vasc. Surg., 53, 1341-1349 e1343.

Libby, P. (2002) Inflammation in atherosclerosis. Nature, 420, 868-874.

Liu, G., Huang, Y., Lu, X., Lu, M., Huang, X., Li, W. \& Jiang, M. (2010) Identification and characteristics of microRNAs with altered expression patterns in a rat model of abdominal aortic aneurysms. Tohoku J. Exp. Med., 222, 187-193.

Liu, X., Cheng, Y., Zhang, S., Lin, Y., Yang, J. \& Zhang, C. (2009) A necessary role of miR-221 and miR-222 in vascular smooth muscle cell proliferation and neointimal hyperplasia. Circ. Res., 104, 476-487.

Livak, K.J. \& Schmittgen, T.D. (2001) Analysis of relative gene expression data using real-time quantitative PCR and the 2(-Delta Delta C(T)) Method. Methods, 25, 402-408.

Lowell, R.C., Gloviczki, P. \& Miller, V.M. (1992) In vitro evaluation of endothelial and smooth muscle function of primary varicose veins. J. Vasc. Surg., 16, 679-686.

Martin, M.M., Buckenberger, J.A., Jiang, J., Malana, G.E., Nuovo, G.J., Chotani, M., Feldman, D.S., Schmittgen, T.D. \& Elton, T.S. (2007) The human angiotensin II type 1 receptor +1166 A/C polymorphism attenuates microrna-155 binding. J. Biol. Chem., 282, 24262-24269.

Meissner, M.H., Gloviczki, P., Bergan, J., Kistner, R.L., Morrison, N., Pannier, F., Pappas, P.J., Rabe, E., Raju, S. \& Villavicencio, J.L. (2007) Primary chronic venous disorders. J. Vasc. Surg., 46 Suppl S, 54S-67S.

O'Connell, R.M., Rao, D.S., Chaudhuri, A.A., Boldin, M.P., Taganov, K.D., Nicoll, J., Paquette, R.L. \& Baltimore, D. (2008) Sustained expression of microRNA-155 in hematopoietic stem cells causes a myeloproliferative disorder. J. Exp. Med., 205, 585-594.

Rabe, E. \& Pannier, F. (2010) Societal costs of chronic venous disease in CEAP C4, C5, C6 disease. Phlebology, 25 Suppl 1, 64-67.

Raffetto, J.D., Vasquez, R., Goodwin, D.G. \& Menzoian, J.O. (2006) Mitogen-activated protein kinase pathway regulates cell proliferation in venous ulcer fibroblasts. Vasc. Endovascular. Surg., 40, 59-66.

Raffetto, J.D. \& Khalil, R.A. (2008) Matrix metalloproteinases in venous tissue remodeling and varicose vein formation. Curr. Vasc. Pharmacol., 6, 158-172.

Rodriguez, A., Vigorito, E., Clare, S., Warren, M.V., Couttet, P., Soond, D.R., van Dongen, S., Grocock, R.J., Das, P.P., Miska, E.A., Vetrie, D., Okkenhaug, K., Enright, A.J., Dougan, G., Turner, M. \& Bradley, A. (2007) Requirement of bic/ microRNA-155 for normal immune function. Science, 316, 608-611.

Ruckley, C.V., Evans, C.J., Allan, P.L., Lee, A.J. \& Fowkes, F.G. (2002) Chronic venous insufficiency: clinical and duplex correlations. The Edinburgh Vein Study of venous disorders in the general population. J. Vasc. Surg., 36, 520-525.

Schlitt, T., Palin, K., Rung, J., Dietmann, S., Lappe, M., Ukkonen, E. \& Brazma, A. (2003) From gene networks to gene function. Genome Res., 13, 2568-2576.

Schrauder, M.G., Strick, R., Schulz-Wendtland, R., Strissel, P.L., Kahmann, L., Loehberg, C.R., Lux, M.P., Jud, S.M., Hartmann, A., Hein, A., Bayer, C.M., Bani, M.R., Richter, S., Adamietz, B.R., Wenkel, E., Rauh, C., Beckmann, M.W. \& Fasching, P.A. (2012) Circulating micro-RNAs as potential blood-based markers for early stage breast cancer detection. PLoS One, 7, e29770.

Tivnan, A., Tracey, L., Buckley, P.G., Alcock, L.C., Davidoff, A.M. \& Stallings, R.L. (2011) MicroRNA-34a is a potent tumor suppressor molecule in vivo in neuroblastoma. BMC Cancer, 11,33 .

Tummala, P.E., Chen, X.L., Sundell, C.L., Laursen, J.B., Hammes, 
C.P., Alexander, R.W., Harrison, D.G. \& Medford, R.M. (1999) Angiotensin II induces vascular cell adhesion molecule-1 expression in rat vasculature: a potential link between the renin-angiotensin system and atherosclerosis. Circulation, 100, 1223-1229.

Vigorito, E., Perks, K.L., Abreu-Goodger, C., Bunting, S., Xiang, Z., Kohlhaas, S., Das, P.P., Miska, E.A., Rodriguez, A., Bradley, A., Smith, K.G., Rada, C., Enright, A.J., Toellner, K.M., Maclennan, I.C. \& Turner, M. (2007) microRNA-155 regulates the generation of immunoglobulin class-switched plasma cells. Immunity, 27, 847-859.

Wang, H., Ach, R.A. \& Curry, B. (2007) Direct and sensitive miRNA profiling from low-input total RNA. RNA, 13, 151-159.

Wang, S., Aurora, A.B., Johnson, B.A., Qi, X., McAnally, J., Hill,
J.A., Richardson, J.A., Bassel-Duby, R. \& Olson, E.N. (2008) The endothelial-specific microRNA miR-126 governs vascular integrity and angiogenesis. Dev. Cell, 15, 261-271.

Yang, W.J., Yang, D.D., Na, S., Sandusky, G.E., Zhang, Q. \& Zhao, G. (2005) Dicer is required for embryonic angiogenesis during mouse development. J. Biol. Chem., 280, 9330-9335.

Yi, M., Horton, J.D., Cohen, J.C., Hobbs, H.H. \& Stephens, R.M. (2006) WholePathwayScope: a comprehensive pathway-based analysis tool for high-throughput data. BMC Bioinformatics, 7,30 .

Yin, H., Zhang, X., Wang, J., Yin, W., Zhang, G., Wang, S. \& Liu, Q. (2006) Downregulation of desmuslin in primary vein incompetence. J. Vasc. Surg., 43, 372-378.

Zhang, C. (2010) MicroRNAs in vascular biology and vascular disease. J. Cardiovasc. Transl. Res., 3, 235-240. 